
\title{
25 Research Soure \\ Impact of the SARS-CoV-2 S-protein G476S mutation on the interaction with human ACE-2 receptor and neutralizing antibodies
}

\section{Alexander Kwarteng ( $\sim$ akwarteng@knust.edu.gh )}

Kwame Nkrumah University of Science and Technology (KNUST)

\section{Ebenezer Asiedu}

Kumasi Centre for Collaborative Research in Tropical Medicine (KCCR)

\section{Augustina Angelina Sylverken}

Kumasi Centre for Collaborative Research in Tropical Medicine (KCCR)

Amma Larbi

Kwame Nkrumah University of Science and Technology (KNUST)

Peter Twumasi

Kwame Nkrumah University of Science and Technology (KNUST)

\section{Research Article}

Keywords: G476S, S-protein, RBD, neutralizing antibody, COVID-19

Posted Date: May 6th, 2021

DOI: https://doi.org/10.21203/rs.3.rs-98463/v3

License: (c) (i) This work is licensed under a Creative Commons Attribution 4.0 International License. Read Full License 


\section{Abstract}

The G476S mutation of the SARS-CoV-2 S-protein occurs in the receptor binding domain (RBD), the region that binds to the human angiotensin-converting enzyme 2 (hACE-2) receptor and also the main target for neutralizing antibodies. The 476S variant was first reported in the USA. Emerging evidence show that the 476 S variant resists neutralization by antibodies such as S2E12 and CC6.29. The impact of the mutation on the interactions with hACE-2 receptor and the dynamics of the S-protein, has not been not fully explored. Here, we provide insights into the structure dynamics of the $476 \mathrm{~S}$ variant and investigate the impact of the mutation on interactions with hACE-2 and selected neutralizing antibodies. We report that the mutation induces a destabilization effect in the RBD and an increased flexibility for most of the receptor binding residues. The mutation, however, does not affect the interactions with the hACE-2 receptor. Both Gly-476 and Ser-476, although located within the hACE-2 interacting residue hotspot, do not contribute to the stabilization of the RBD-hACE-2 complex. Our findings suggest that both H014 and P2P2F6 antibodies neutralize the $476 \mathrm{G}$ and $476 \mathrm{~S}$ S-proteins with similar efficacy.

\section{Introduction}

The coronavirus disease 2019 (COVID-19), which has been a global threat since November 2019 is caused by the severe acute respiratory syndrome coronavirus-2 (SARS-CoV-2). Symptoms at the early stages of the disease include fever, fatigue and dry $\operatorname{cough}^{1,2}$, but can progress to a severe state with symptoms of pneumonia and severe complications ${ }^{3}$. SARS-CoV-2 is an enveloped positive-strand RNA virus belonging to the genus Betacoronavirus ${ }^{4}$. The SARS-CoV-2 infection is initiated when the viral transmembrane spike (S) glycoprotein (S-protein) binds to the human angiotensin-converting enzyme 2 (hACE-2) receptor through a receptor-binding domain (RBD), causing membrane fusion and entry into host cells $s^{6,7}$. The RBD of the S-protein is also the major target for the robust host immune responses elicited against the virus, including neutralizing antibodies ${ }^{8}$. Neutralizing antibodies bind to the receptorbinding domain (RBD) of the S-protein in order to disrupt interactions between the S-protein and the receptor protein, preventing viral entry into host cells and subsequent infection ${ }^{9-11}$.

Recently, several variations of interest of the SARS-CoV-2 have been reported, a phenomenon common to RNA viruses ${ }^{12}$. The $614 \mathrm{G}$ variant, which became the dominant circulating strain globally, was described to favor the open conformation state of the S-protein, resulting in increased transmissibility and higher viral loads in patients ${ }^{13,14}$. In addition, the $480 \mathrm{~A} / \mathrm{G}$ variants of the SARS-CoV-1 S-protein have been reported to escape neutralization by the antibody $80 \mathrm{R}^{15}$. The current circulating strains of high concern (B.1.1.7, B.1.351 and P.1) contains mutations such as $\mathrm{N} 501 \mathrm{Y}, \mathrm{E} 484 \mathrm{~K}$ and $\mathrm{K} 417 \mathrm{~N} / \mathrm{T}^{16-18}$. Emerging evidence show that the sensitivity to antibody neutralization vary for these variants ${ }^{16-18}$.

The 476 S variants was first reported in the USA and persisted for over 11 weeks ${ }^{19,20}$. This mutation, like those in the strains of concern, occurs in the RBD, the region responsible for hACE-2 binding and also the main target for neutralizing antibody binding. The G476S mutation attenuates a linear and a 
discontinuous B-cell epitope in the $\mathrm{RBD}^{21}$, which could be a possible escape mechanism to host immune responses. Other studies have shown that the $476 \mathrm{~S}$ variant resist neutralization by antibodies such as $\mathrm{S} 2 \mathrm{E} 12^{22}$ and $\mathrm{CC} 6.29^{23}$.

The impact of the mutation on interactions between the S-protein and hACE-2 receptor, has not been fully explored yet. It is also unclear whether the mutation has an impact on the molecular dynamics of the Sprotein. To provide insights into these issues, the present study aimed to investigate the influence of the mutation (G476S) on the interactions between the SARS-CoV-2 S-protein and hACE-2 receptor as well as some selected neutralizing antibodies (H014 and P2P-2F6). We further aimed to study how the mutation affect the structure dynamics of the S-protein.

\section{Method}

\section{Building and Validating Protein Systems}

The sequence of the SARS-CoV-2 S-protein belonging to the Wuhan Reference sequence was obtained from the UniProt database (https://www.uniprot.org/uniprot/A0A679G9E9). We also retrieved the human ACE-2 sequence from the same database. We built tertiary structures of the proteins using the SWISSMODEL tool ${ }^{24}$. The S-protein and hACE-2 models were generated using the crystallographic coordinates of PDB entries 7DK3 and 6LZG, respectively. SWISS-MODEL is a homology modeling tool that relies on the OpenStructure computational structure biology framework ${ }^{25}$ and ProMod $3^{26}$ modeling engine to build protein structures based on coordinates of templates ${ }^{24}$. Moreover, the tool checks modeling errors and assess structure quality using the qualitative model energy analysis (QMEAN) scoring function ${ }^{27}$. SWISSMODEL ranks among the top-performing modeling servers ${ }^{24}$. All structure quality assessments were performed with the SWISS-MODEL quality assessment tool. Having confirmed the suitability of the protein models, we generated the $476 \mathrm{~S}$ variant from the wild-type S-protein structure using the PyMol v2. ${ }^{28}$ mutagenesis function. For the antibody-S-protein complexes, we superimposed the S-protein model to the PDB entries of the antibodies and removed the S-protein in the template. The H014 and P2B2F6 templates were PDB entries 7CAK and 7BWJ.

\section{Determining the degree of evolutionary conservation of S-protein residues}

The S-protein structure was submitted to the ConSurf too ${ }^{29}$ to calculate the degree of evolutionary conservation of the SARS-CoV-2 S-protein residues. ConSurf is widely used to determine the evolutionary dynamics of protein or nucleic acid residues. The algorithm performs BLAST searches of query inputs (amino acids or nucleic acid residues) against the UNIREF-90 database and generate phylogenetic trees based on derived multiple sequence alignment ${ }^{29}$. Based on an empirical Bayesian methodology, ConSurf uses the Rate4Site algorithm ${ }^{31}$ to compute evolutionary rates of each residue. These rates are normalized and ranked into grades from 1 (highly variable residue) to 9 (highly conserved residue). We used the CSIBLAST as homologous search algorithm and a total of three number of iterations. The E-value cut-off was 0.0001 . Total number of reference sequences to be used was 150 with $95 \%$ maximum sequence 
identity. The minimum identity for counterparts was set to $35 \%$. The default values for alignment method, calculation method and evolutionary substitution model were used.

\section{Simulating the molecular dynamics of protein systems}

All simulations were performed with GROMACS ${ }^{31}$ version 2021. The parameters from the CHARMM $36^{32}$ force field and TIP3P water model were used in all systems. To mimic the full length of both S-protein and hACE-2 receptor, we capped the $\mathrm{N}$-termini and $\mathrm{C}$-termini with acetyl group and $\mathrm{N}$-methyl amide, respectively. The canonical states of all titrable amino acids were assigned at physiological $\mathrm{pH}$. Van derWaals interactions were treated with a single cut-off of $1.4 \mathrm{~nm}$. Long-range electrostatics were treated with an advanced Particle-Particle Particle-Mesh algorithm (P3M-AD) and a cut-off of $1.4 \mathrm{~nm}$. Neighbor searching was performed every 10 steps. We also applied periodic boundaries in all directions.

Protein systems were placed in a cubic box such they were $2 \mathrm{~nm}$ from the box edges. The systems were fully solvated and neutralizing counter-ions $(\mathrm{NaCl})$ were added. To obtain a suitable starting structure for the simulation, the systems were energy minimized using the steepest descent algorithm for 20,000 steps. We then simulated the proteins systems with position restraints on heavy atoms under constant volume (NVT) ensemble for 400 ps and constant pressure (NPT) ensemble for 400 ps. Protein and nonprotein components of the systems were independently coupled to V-rescale thermostat and isotropic Berendsen algorithm for pressure coupling. For molecular simulation analysis, production simulations were performed for $100 \mathrm{~ns}$ in triplicates without any constraints at a time step of $2 \mathrm{fs}$. Simulations for binding energy analysis were performed for $15 \mathrm{~ns}$ in triplicates. A summary of the simulation and protein systems is provided in Table 1.

Table 1

Details of molecular simulation systems. Fab = Antigen binding fragment.

\begin{tabular}{|lll|}
\hline Molecular system & Simulation time (ns) & Repeats \\
\hline Molecular dynamics analysis & & \\
\hline wild-type S-protein monomer & 100 & 3 \\
\hline 476S variant S-protein monomer & 100 & 3 \\
\hline MM-PBSA binding energy analysis & 3 \\
\hline wild-hACE2 complex & 15 & 3 \\
\hline 476S-hACE-2 complex & 15 & 3 \\
\hline wild-H014 Fab complex & 15 & 3 \\
\hline 476S-H014 Fab complex & 15 & 3 \\
\hline wild-P2B-2F6 Fab complex & 15 & 3 \\
\hline 476S-P2B-2F6 Fab complex & 15 & 3 \\
\hline
\end{tabular}

Page 4/19 


\section{Trajectory preparations and MD analysis}

All trajectory preparations, including re-centering, fitting, periodicity treatments, and concatenation of the trajectories, were performed with in-built GROMACS tools. The dynamics of molecular systems were assessed using root-mean-square deviation (RMSD) and root-mean-square fluctuation (RMSF). The RMSD was calculated over all backbone atoms after least-squares fit to the reference backbone, while the RMSF was calculated per residue after least-squares fit to C-a atoms. All graphical representations were generated with GraphPad Prism v8.0. RMSD and RMSF plots were averaged over three independent simulations. Molecular visualizations of structures were done with PyMol v2.4 ${ }^{28}$.

\section{Binding energy calculations}

The binding energy and individuals forces of interactions were calculated with g_mmpbsa v5.1.2 ${ }^{33}$. The tool implements the molecular mechanics with Poisson-Boltzmann surface area (MM-PBSA) calculations on structural frames derived from GROMACS simulations trajectories. Details of the molecular systems used for MM-PBSA calculations are provided in Table 1. For each complex, calculations were performed on 225 frames sampled over three independent $15 \mathrm{~ns}$ simulation. Binding energy and interaction energies were computed as previously described ${ }^{33}$. Briefly, the total binding energy is the summation of four individual energy terms; van der Waals, electrostatics, polar solvation and non-polar solvation energies. The current g_mmpbsa free energy of binding. The tool is however appropriate for measuring relative binding energies, such as when comparing different ligands/proteins that bind to the same receptor. The binding energy, $\mathrm{E}_{\text {binding }}$ is represented as;

$E_{\text {binding }}=\left(E_{\mathrm{vdW}}+E_{\text {elec }}\right)+\left(G_{\text {polar }}+G_{\text {nonpolar }}\right)$

where the bonded interactions $\left(E_{\text {bonded }}\right)$ consist of bond, angle, dihedral and improper interactions. The non-bonded interactions include electrostatic $\left(\mathrm{E}_{\mathrm{elec}}\right)$ and van der Waals $\left(\mathrm{E}_{\mathrm{vdW}}\right)$ interaction forces which are calculated using Coulomb and Lennard-Jones potential functions, respectively. The polar solvation does not calculate contributions from entropic terms, i.e. does not give the absolute energy $\left(G_{\text {polar }}\right)$, which is the electrostatic contribution to solvation is computed using the Poisson-Boltzmann equation. The nonelectrostatic contribution to the solvation energy, which is estimated from the solvent accessible surface area (SASA) is represented by the non-polar energy $\left(G_{\text {nonpolar }}\right)$. Parameters for calculating energy terms and values for constants were adopted from a previous study ${ }^{33}$.

\section{Results}

\section{Validation of the SARS-CoV-2 and human ACE-2 models}

All models were built and validated using the SWISS-MODEL Homology modeling tool and the SWISSMODEL quality assessment tool, respectively. For this study, the Chain A monomer of the SARS-CoV-2 Sprotein in the open state conformation was used for all analysis. The hACE-2 and the selected 
neutralizing antibodies all interact with the S-protein in the open state. The S-protein and hACE-2 models were generated using the crystallographic coordinates of PDB entries 7DK3 and 6LZG, respectively. The global model quality estimation (GMQE) and qualitative model energy analysis (QMEAN) scores were used to decide the model selection. Ramachandran plot of each protein model was generated to confirm their quality (Fig. 1a). Comparison of the built models to a set of non-redundant protein structures of high quality in the PDB database also showed that the models were suitable for this study (Fig. 1b). We then performed a pair-wise alignment of the model structure and their respective templates. The alignments of the model-template structures are shown in Fig. 1c. Having assessed the suitability of the model for this study, we used the PyMol v2.4 mutagenesis tool to create the $476 \mathrm{~S}$ variant by replacing Gly-476 with Ser476 in the RBD (Fig. 1d).

\section{Effect of the mutation on RBD structure dynamics}

To determine the impact of the mutation on S-protein structure dynamics, we first characterized the degree of evolutionary conservation of Gly-476 using the ConSurf tool ${ }^{29}$. Usually, the natural tendency of an amino acid mutating largely depends on its importance to the protein's structure and functions. The ConSurf conservation profile for the S-protein show that most of the conserved residues are in the S2subunit, with the most variable residues in the RBD and the NTD (Fig. 2). The profile shows a ConSurf score of 1 for Gly-476, suggesting that it is highly variable and easily mutable.

To assess whether G476S mutation induces any conformation change in the S-protein, we used rootmean-square deviation (RMSD), RMSD distribution and root-mean-square fluctuation (RMSF) to compare the structural dynamics of the wild-type and 476S variant S-proteins (Fig. 3a-3e). The RMSD describes the conformation stability of the protein system during the simulation by indicating the average displacement of backbone atoms in the protein structure with respect to the starting structure (equilibrated structure, $t=0 \mathrm{~ns}$ ). The backbone-RMSD value of the wild-type S-protein averaged $1.08 \mathrm{~nm}$, which increased to $1.18 \mathrm{~nm}$ in the case of the $476 \mathrm{~S}$ variant S-protein, indicating a destabilization impact of the mutation (Fig. 3a). To determine the most affected regions of the S-proteins, domain-specific backbone-RMSD profiles were generated (Fig. 3b). The destabilization effect of the mutation was largely observed in the RDB than the NTD and the S2-subunit. The backbone-RMSD of the wild-type RBD averaged $0.39 \mathrm{~nm}$, which increased to $0.45 \mathrm{~nm}$ for the $476 \mathrm{~S}$ variant RBD.

The structures from the simulation trajectories were clustered using RMSD cut-off of $0.1 \mathrm{~nm}$ and the gromos method, resulting in several cluster groups. The group-centre structure of the most populated cluster for both wild-type and $476 \mathrm{~S}$ variant S-proteins were selected and superimposed. The structure superposition shows that the wild-type and 476S variant S-proteins differ by RMSD of $5.3 \AA$ (Fig. 3d). The difference in the RBD is also emphasized. The increased backbone-RMSD of the $476 \mathrm{~S}$ variant can be attributed to destabilization effect of the mutation which is most pronounced in the RBD.

The residue-specific flexibility of protein structure is indicated by the RMSF. The RMSF was calculated to evaluate the residue flexibility to compare the residue-by-residue variations between the wild-type and 
476S S-proteins. Although both the systems assumed a similar residue flexibility profile, the residue fluctuations were higher in the $476 \mathrm{~S}$ variant RBD, compared to the wild-type RBD (Fig. 3e). The most affected regions ranged from residues $347-350,370-393,413-428,456-459$ and $471-487$. This further indicates the destabilization effect of the mutation in the RBD, resulting in increased flexibility for most of the local residues.

\section{Impact of the mutation on stabilization of the RBD-hACE-2 complex}

To investigate the potential impact of the mutation on the interaction between the S-protein and hACE-2 receptor, we first examined the dynamics of the RBD upon binding to the hACE-2 receptor. Comparing RMSD of the RBD in bound and unbound states, we observed that the RBD was destabilized upon binding to the hACE-2 receptor (Fig. 4a), for both the wild-type and 476S variant RBD-hACE-2 complexes. Further, the residue-specific flexibility was significantly affected upon binding to hACE-2, most especially in the RBD. The residues in the RBD demonstrated less flexibility upon binding to the hACE-2 receptor (Fig. 4b). The most affected regions were residues 330-395 and the least affected region ranged from residues $473-503$, including Gly-476 and Ser-476. This suggests a major participation in the interactions with hACE-2 for the residues 330-395.

We examined the interacting interface of the RBD-hACE-2 complex to determine whether Gly-476 and Ser476 were located within the hACE-2 interacting residue hotspot during the simulation. The middlestructure of the most populated cluster group was selected for this activity. All amino acids that are within $5 \AA$ of the hACE-2 receptor were considered, assuming that most forces of interaction are captured within this distance. Both Gly-476 and Ser-476 were located in the interacting residue hotspot of the RBDhACE-2 complex during the simulation (Fig. 5).

To characterize how the mutation affect RBD-hACE-2 interactions, the binding energy and the individual energy terms governing the RBD-hACE-2 stabilization were calculated. The energy terms for each complex were calculated using 225 structure frames sampled over three independent $15 \mathrm{~ns}$ simulations. The estimated binding energies were highly comparable for the wild-type $(-2068.268 \pm 138.127 \mathrm{~kJ} / \mathrm{mol})$ and $476 \mathrm{~S}$ variant $(-1953.245 \pm 128.816 \mathrm{~kJ} / \mathrm{mol})$, suggesting that the interactions with hACE- 2 is not largely affected by the G476S mutation in the RBD. The computed individual energy terms governing the complex stabilization also show high similarity for the wild-type and 476S variant complexes (Table 2). 
Table 2

Energy terms governing the interactions between the RBD and human

ACE-2 receptor.

\begin{tabular}{|lll|}
\hline & \multicolumn{2}{l|}{ RBD-hACE-2 interaction energy $(\mathrm{kJ} / \mathrm{mol})$} \\
\hline Energy term & wild-type & $476 S$ variant \\
\hline van der Waal & $-673.925 \pm 128.840$ & $-555.784 \pm 85.479$ \\
\hline Electrostatics & $-3186.796 \pm 224.890$ & $-2860.553 \pm 164.651$ \\
\hline Polar solvation & $1871.273 \pm 273.396$ & $1527.539 \pm 179.273$ \\
\hline Non-polar solvation & $-78.820 \pm 11.486$ & $-64.447 \pm 8.023$ \\
\hline Total energy & $-2068.268 \pm 138.127$ & $-1953.245 \pm 128.816$ \\
\hline
\end{tabular}

To determine the contribution of the Gly-476 and Ser-476 to the overall binding energy, we computed the residue contribution to the stabilization of the interaction between the S-protein and hACE-2. The energy contribution profile of the RBD residues are shown in Fig. 6a. The contributions of Gly-476 and Ser-476 to the overall stabilization of the RBD-hACE-2 complexes were $-5.7 \mathrm{~kJ} / \mathrm{mol}$ and $-2.7 \mathrm{~kJ} / \mathrm{mol}$, respectively. These contributions are insignificant to the overall stabilization of the RBD-hACE-2 complexes. Based on their energy contributions, the residues ranging from 475-503, play no significant roles in the RBD-hACE2 interactions. The largest contributors to the complex stabilization were from residues 346-424. Although Gly-476 is replaced with a polar residue in the $476 \mathrm{~S}$ variant, individual energy terms such as electrostatic and van der Waals energy contributions were larger in the wild-hACE-2 complex than the 476S-hACE-2 complex. We, therefore, generated a distance plot for the Gly-476/Ser-476 and hACE-2. The minimum distance between the hACE-2 receptor and Gly-476 averaged $0.23 \mathrm{~nm}$, which increased to 0.43 nm for Ser-476 (Fig. 6b).

\section{Impact of the mutation on interactions with $\mathrm{H} 014$ and P2B-2F6}

Previous studies have established that the $476 \mathrm{~S}$ variant resists neutralization by particular antibodies such as S2E12 $2^{22}$ and CC6.2923. Thus, we studied the impact of the mutation on interactions with two neutralization antibodies that target the RBD but have different mechanisms of neutralization. The antibodies $\mathrm{H} 014$ and P2B-2F6 were selected for this particular study. Although both antibodies target the RBD, H014 neutralizes by inducing sterical clashes to the RBD and interfere with hACE-2 binding ${ }^{34}$. P2B2F6, however, directly competes with the RBD for hACE-2 binding ${ }^{35}$ (Fig. 7a).

Analysis of the individual residue contributions to the complex interactions revealed that both Gly-476 and Ser-476 have insignificant contributions to the stabilization of both RBD-H014 and RBD-H014 complexes (Fig. 7b). Moreover, residue contributions for both RBD-H014 and RBD-P2B-2F6 complexes were similar for the wild-type and $476 \mathrm{~S}$ variant. The energy terms governing the RBD and neutralizing antibody interactions are summarized in Table 3 . The estimated binding energy and individual energy terms did not vary significantly between the wild-type and $476 \mathrm{~S}$ variant interactions with the neutralizing 
antibodies. These suggest that the mutation has no effect on the interactions between the RBD and the neutralizing antibodies studied.

Table 3

Energy terms governing the interactions between the RBD and neutralizing antibodies ( $\mathrm{H} 014$ and P2B2F6).

\begin{tabular}{|c|c|c|c|c|}
\hline \multirow[b]{2}{*}{ Energy } & \multicolumn{2}{|c|}{ RBD-H014 interaction energy $(\mathrm{kJ} / \mathrm{mol})$} & \multicolumn{2}{|c|}{$\begin{array}{l}\text { RBD-P2B-2F6 interaction energy } \\
(\mathrm{kJ} / \mathrm{mol})\end{array}$} \\
\hline & wild-type & $476 S$ variant & wild-type & $476 \mathrm{~S}$ variant \\
\hline van der Waal & $-634.710 \pm 69.288$ & $-627.918 \pm 55.067$ & $\begin{array}{l}-409.078 \pm \\
62.653\end{array}$ & $\begin{array}{l}-465.034 \pm \\
55.456\end{array}$ \\
\hline Electrostatics & $\begin{array}{l}-1019.975 \pm \\
156.196\end{array}$ & $\begin{array}{l}-1108.485 \pm \\
136.660\end{array}$ & $\begin{array}{l}109.956 \pm \\
30.520\end{array}$ & $91.482 \pm 132.519$ \\
\hline Polar solvation & $\begin{array}{l}1297.268 \pm \\
195.574\end{array}$ & $\begin{array}{l}1370.605 \pm \\
142.078\end{array}$ & $\begin{array}{l}660.597 \pm \\
86.951\end{array}$ & $\begin{array}{l}861.206 \pm \\
149.536\end{array}$ \\
\hline $\begin{array}{l}\text { Non-polar } \\
\text { solvation }\end{array}$ & $-67.686 \pm 4.429$ & $-69.454 \pm 4.976$ & $-46.876 \pm 5.749$ & $-53.460 \pm 4.034$ \\
\hline Total energy & $\begin{array}{l}-425.103 \pm \\
106.969\end{array}$ & $\begin{array}{l}-435.252 \pm \\
101.365\end{array}$ & $\begin{array}{l}394.599 \pm \\
83.723\end{array}$ & $434.194 \pm 77.513$ \\
\hline
\end{tabular}

\section{Discussion}

Here, we investigated the structure dynamics of the $476 \mathrm{~S}$ variant of SARS-CoV-2 and characterize the features of interaction with hACE-2 receptor and some selected neutralizing antibodies. We report that the $476 S$ variant has a destabilized RBD and increased residue-specific flexibility in the domain when compared to the wild-type. Despite the observed changes in conformation particularly in the RBD, interactions with hACE-2 were not significantly affected by the mutation. The wild-type and $476 \mathrm{~S}$ variant RBDs interacted with the hACE-2 receptor with highly similar interaction energies. We studied the Sproteins complexed to the antigen binding fragments of neutralizing antibodies $\mathrm{H} 014$ and P2B-2F6. Our results suggest that both antibodies have equivalent neutralizing potency to the $476 \mathrm{G}$ and $476 \mathrm{~S}$ Sproteins.

The G476S mutation occurs within a cluster of amino acids that define the receptor interacting interface in the RBD ${ }^{36}$. The current virus strains that are classified as variants of interest contain mutations such as N501Y and E484K, all occurring in the said cluster of amino acids. Usually, the tendency of a residue mutating largely depends on its degree of evolutionary conservation. The ConSurf evolutionary conservation profile show that the RBD residues that interact with the hACE-2 receptor are highly variable with a high tendency of mutation.

Despite the replacement of Gly-476 with a more polar serine residue in the RBD, the interactions with the hACE-2 receptor were not affected significantly. Particularly, both Gly-476 and Ser-476 were not critical to 
the stabilization of the RBD-hACE-2 complex. We observed that RBD residues ranging from 473-503 were least affected in terms of residue-specific flexibility, upon binding to the hACE-2. The same cluster of residues, including residue 476 , were the least contributors to the stabilization of RBD-hACE-2 complex.

The G476S mutation attenuates a linear and a discontinuous B-cell epitope in the RBD ${ }^{21}$, which could be a possible escape mechanism to host immune responses. The $476 \mathrm{~S}$ has also been shown to resist antibody neutralization by S2E $12^{22}$ and $\mathrm{CC} 6.29^{23}$. We studied the S-proteins complexed to the antigen binding fragments of neutralizing antibodies $\mathrm{H} 014$ and $\mathrm{P} 2 \mathrm{~B}-2 \mathrm{~F} 6$. The mutation had no significant impact on the interactions with the antibodies. Both Gly-476 and Ser-476 provided no contributions to the interactions between the RBD and the antibodies. Thus, resistance of antibody neutralizing by the $476 \mathrm{~S}$ variant largely depends on the contribution of the Ser-476 to the antibody-RBD interactions and the epitopes recognized by the antibody.

There could be several variants with mutations in the RBD having mechanisms of antibody neutralizing resistance or improved virus survival but are present at low frequencies in SARS-CoV-2 infected populations ${ }^{37}$. The findings of this study suggest that the binding of the S-protein RBD, H014 Fab and P2B-2F6 Fab to the hACE-2 receptor are not affected by the mutation. However, previous reports suggest that the $476 \mathrm{~S}$ variant has mechanisms of escaping the host immunity. Robust surveillance should be performed to track the distribution and spread of the $476 \mathrm{~S}$ variant.

\section{Conclusion}

In this study, we investigated the structure dynamics of the 476S variant of SARS-CoV-2 S-protein, and describe the features of interaction with the hACE-2 receptor and selected neutralizing antibodies. Compared to the wild-type, the $476 \mathrm{~S}$ variant has a destabilized RBD and high residue-specific flexibility in the domain. Despite the observed changes in conformation, especially in the RBD, the mutation had no effect on interactions with hACE-2. The wild-type and 476S variant RBDs had very similar energies when they interacted with the hACE-2 receptor. S-proteins bound to antigen binding fragments of neutralizing antibodies $\mathrm{H} 014$ and P2B-2F6 were also studied. Our findings suggest that both antibodies neutralize the $476 \mathrm{G}$ and $476 \mathrm{~S}$ S-proteins with similar efficacy.

\section{Declarations}

Author Contribution

Concept: AK, EA, AS

Methodology: AK, EA

Manuscript Writing: AK, EA, AS

Review \& Editing: AK, EA, AS

Page $10 / 19$ 


\section{Funding}

This research did not receive funding.

\section{$\underline{\text { Conflict of interest }}$}

All authors have no conflict of interest.

\section{References}

1. Wang, D. et al. Clinical characteristics of 138 hospitalized patients with 2019 novel coronavirusinfected pneumonia in Wuhan, China. JAMA. 323, 1061-1069 https://doi.org/10.1001/jama.2020.1585 (2020). )

2. Huang, C. et al. Clinical features of patients infected with 2019 novel coronavirus in Wuhan, China. Lancet. 395 (10223), 497-506 https://doi.org/10.1016/j.chom.2020.02.001 (2020). )

3. Ortiz-Prado, D. et al. Clinical, molecular and epidemiological characterization of the SARS-CoV2 virus and the Coronavirus disease 2019 (COVID-19), a comprehensive literature review. Diagnostic Microbiology and Infectious Disease,. https://doi.org/10.1016/j.diagmicrobio.2020.115094 (2020). )

4. Lu, R. et al. Genomic characterisation and epidemiology of 2019 novel coronavirus: implications for virus origins and receptor binding. Lancet. 395, 565-574 (2020). )

5. Naqvi, A. A. T. et al. Insights into SARS-CoV-2 genome, structure, evolution, pathogenesis and therapies: Structural genomics approach. Biochimica et Biophysica Acta (BBA) - Molecular Basis of Disease,(2020). https://doi.org/10.1016/j.bbadis.2020.165878

6. Wan, Y. et al. Receptor recognition by novel coronavirus from Wuhan: an analysis based on decadelong structural studies of SARS. J. Virol. 94, e00127-20 ( (2020). )

7. Walls, A. C. et al. Unexpected Receptor Functional Mimicry Elucidates Activation of Coronavirus Fusion. Cell. 176, 1026-103915 (2019).

8. Jiang, S., Hillyer, C. \& Du, L. Neutralizing Antibodies against SARS-CoV-2 and Other Human Coronaviruses. Trends Immunol. 41, 355-359 (2020).

9. Lv, Z. et al. Structural basis for neutralization of SARS-CoV-2 and SARS-CoV by a potent therapeutic antibody. Science. 5881, eabc5881 (2020).

10. Ju, B. et al. Human neutralizing antibodies elicited by SARS-CoV-2 infection.Nature584, (2020).

11. Hansen, J. et al. Studies in humanized mice and convalescent humans yield a SARS-CoV-2 antibody cocktail. Science. 0827, eabd0827 (2020).

12. Duffy, S. Why are RNA virus mutation rates so damn high? PLoS Biol. 16, e3000003 (2018).

13. Korber, B. et al. Tracking Changes in SARS-CoV-2 Spike: Evidence that D614G Increases Infectivity of the COVID-19 Virus. Cell. 182, 812-82719 (2020). )

14. Mansbach, R. A. et al. The SARS-CoV-2 Spike Variant D614G Favors an Open Conformational State. bioRxiv(2020) doi:10.1101/2020.07.26.219741. 
15. Sui, J. et al. Broadening of neutralization activity to directly block a dominant antibody-driven SARScoronavirus evolution pathway. PLoS Pathog. 4, e1000197 ( (2008). )

16. Wang, P. et al. Antibody Resistance of SARS-CoV-2 Variants B.1.351 and B.1.1.7. Nature 2021, (2021).

17. Egziabher, T. B. G. \& Edwards, S. Sensitivity of infectious SARS-CoV-2 B.1.1.7 and B.1.351 variants to neutralizing antibodies. Nat. Med. 53, 1689-1699 (2013).

18. Collier, D. A. et al. Sensitivity of SARS-CoV-2 B.1.1.7 to mRNA vaccine-elicited antibodies. Nature. https://doi.org/10.1038/s41586-021-03412-7 (2021).

19. Kaushal, N. et al. Mutational Frequencies of SARS-CoV-2 Genome during the Beginning Months of the Outbreak in USA.Pathog. (Basel, Switzerland)9, (2020).

20. Mohammad, S. et al. Exploring the genomic and proteomic variations of SARS-CoV-2 spike glycoprotein: A computational biology approach. Infection, Genetics and Evolution. 84, 104389 (2020).

21. Xu, W., Wang, M., Yu, D. \& Zhang, X. Variations in SARS-CoV-2 Spike Protein Cell Epitopes and Glycosylation Profiles During Global Transmission Course of COVID-19. Front. Immunol. 11, 565278 (2020).

22. Tortorici, M. A. et al. Ultrapotent human antibodies protect against SARS-CoV-2 challenge via multiple mechanisms. 3354,29-31(2020).

23. Rogers, T. F. et al. Isolation of potent SARS-CoV-2 neutralizing antibodies and protection from disease in a small animal model. 7520,1-12(2020).

24. Waterhouse, A. et al. SWISS-MODEL: homology modelling of protein structures and complexes. 46, 296-303 (2018).

25. Biasini, M. et al. OpenStructure: an integrated software framework for computational structural biology. Acta Crystallogr. D, Biol. Crystallogr., 69, 701-709 (2013). )

26. Studer, G. et al. ProMod3-A versatile homology modelling toolbox. PLoS Comput. Biol. 17 (1), e1008667 https://doi.org/10.1371/journal.pcbi.1008667 (2021). )

27. Benkert, P. et al. Toward the estimation of the absolute quality of individual protein structure models. Bioinformatics. 27, 343-350 (2011). )

28. Yuan, S., Chan, H. C. S. \& Hu, Z. Using PyMOL as a platform for computational drug design. Wiley Interdiscip. Rev. Comput. Mol. Sci. 7, 1-10 (2017).

29. Ashkenazy, H. et al. ConSurf 2016: an improved methodology to estimate and visualize evolutionary conservation in macromolecules. Nucleic Acids Res. 44, W344-W350 (2016).

30. Pupko, T., Bell, R. E., Mayrose, I., Glaser, F. \& Ben-Tal, N. Rate4Site: an algorithmic tool for the identification of functional regions in proteins by surface mapping of evolutionary determinants within their homologues. Bioinformatics. 18 (Suppl 1), S71-7 (2002).

31. Abraham, M. J. et al. High performance molecular simulations through multi-level parallelism from laptops to supercomputers. SoftwareX. 1-2, 19-25 (2015). Gromacs 
32. Pastor, R. W. \& MacKerell, A. D. Development of the CHARMM Force Field for Lipids. J. Phys. Chem. Lett. 2, 1526-1532 (2011).

33. Kumari, R., Kumar, R., Lynn, A. \& G-mmpbsa - A GROMACS tool for high-throughput MM-PBSA calculations. J. Chem. Inf. Model. 54, 1951-1962 (2014).

34. Lv, Z. et al. Structural basis for neutralization of SARS-CoV-2 and SARS-CoV by a potent therapeutic antibody. 5881,1-10(2020).

35. Ju, B. et al. Human neutralizing antibodies elicited by SARS-CoV-2 infection. Nature. 584, 115-119 (2020).

36. Yi, C. et al. Key residues of the receptor binding motif in the spike protein of SARS-CoV-2 that interact with ACE2 and neutralizing antibodies. Cell. Mol. Immunol. https://doi.org/10.1038/s41423-0200458-z (2020).

37. Weisblum, Y. et al. Escape from neutralizing antibodies by SARS-CoV-2 spike protein variants. eLife. 9, https://doi.org/10.7554/eLife.61312 (2020).

Figures

(a)
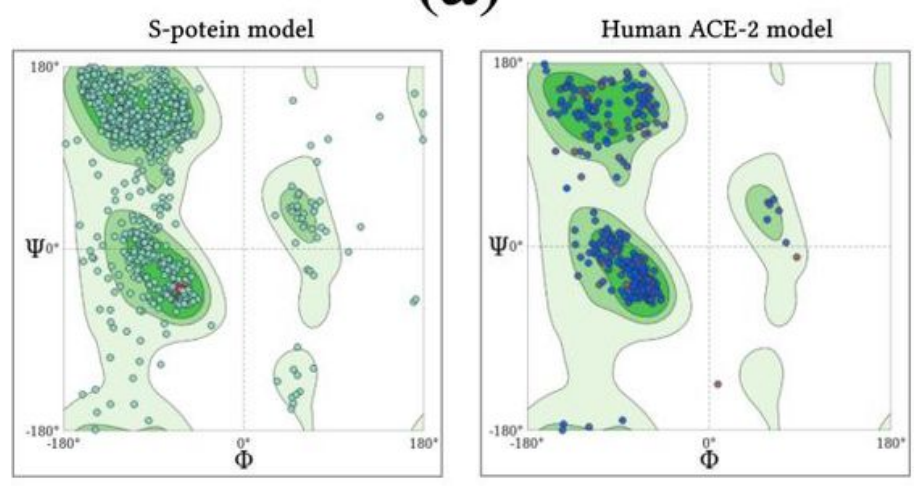

(c)

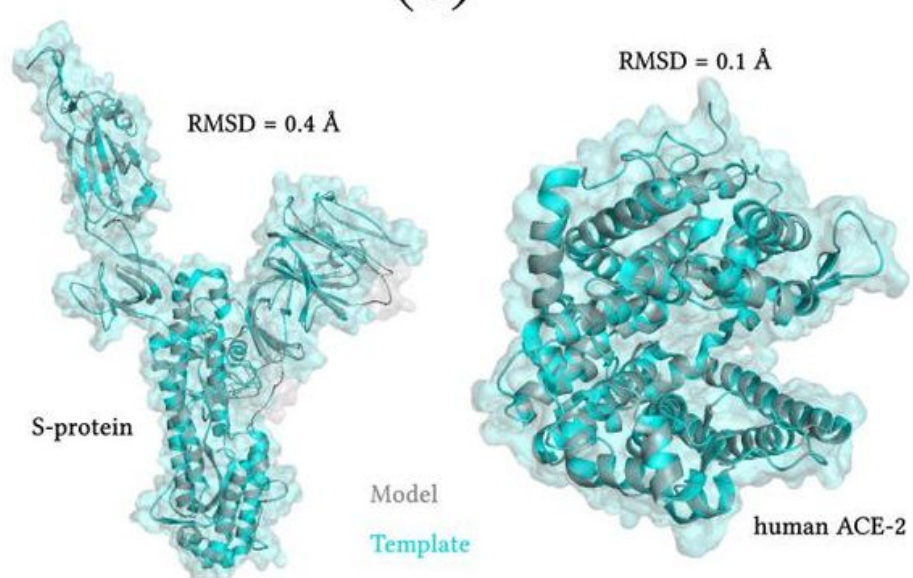

(b)

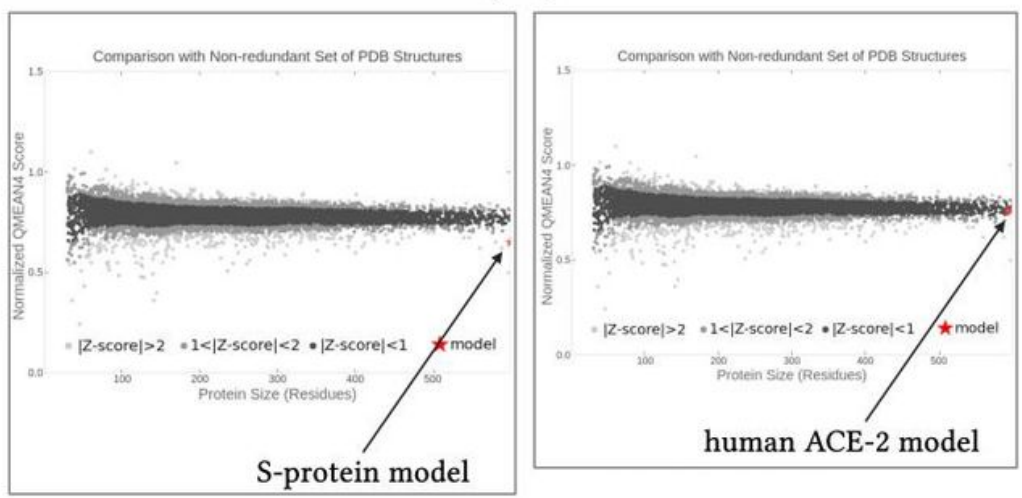

(d)

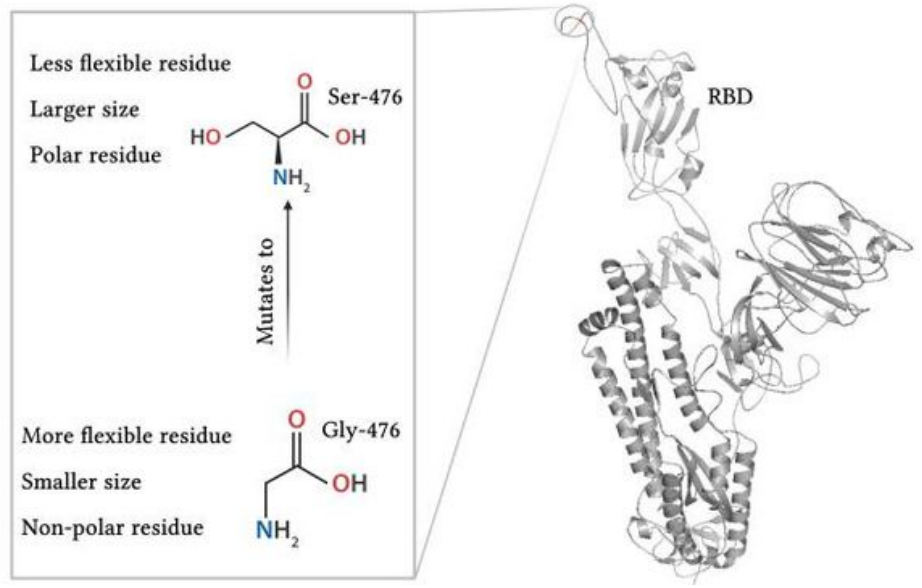

Figure 1 
Validation of SARS-CoV-2 and human ACE-2 models. (a) Ramachandran plots of the models. The plot of the S-protein was based on the Chain A monomer. (b) Comparison of models to non-redundant quality structures at the protein data bank. (c) Structural superposition of the models to their respective templates and the corresponding RMSD. (d) Properties of Gly-476 and Ser-476.

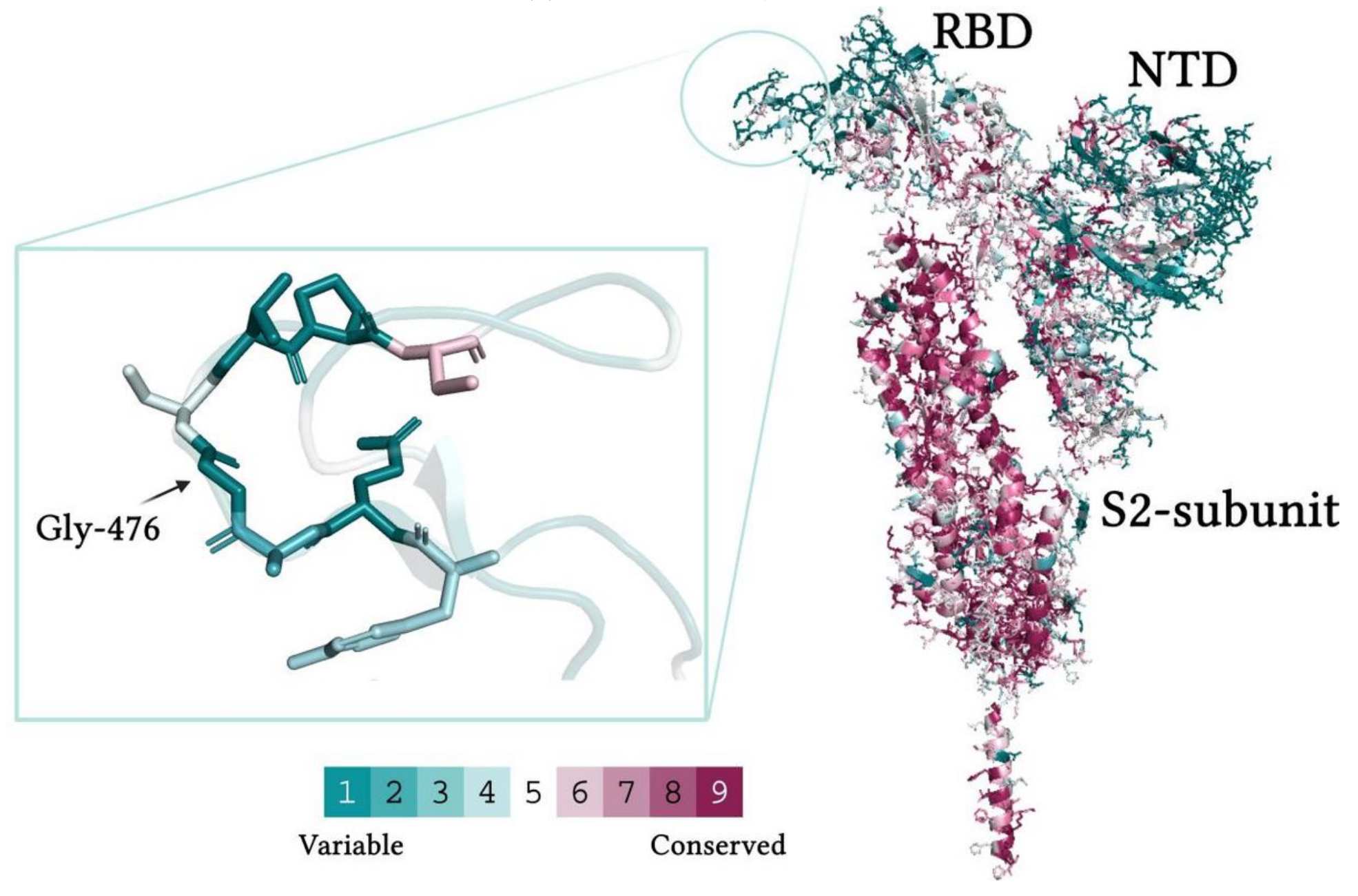

Figure 2

The degree of evolutionary conservation of Gly-476. 

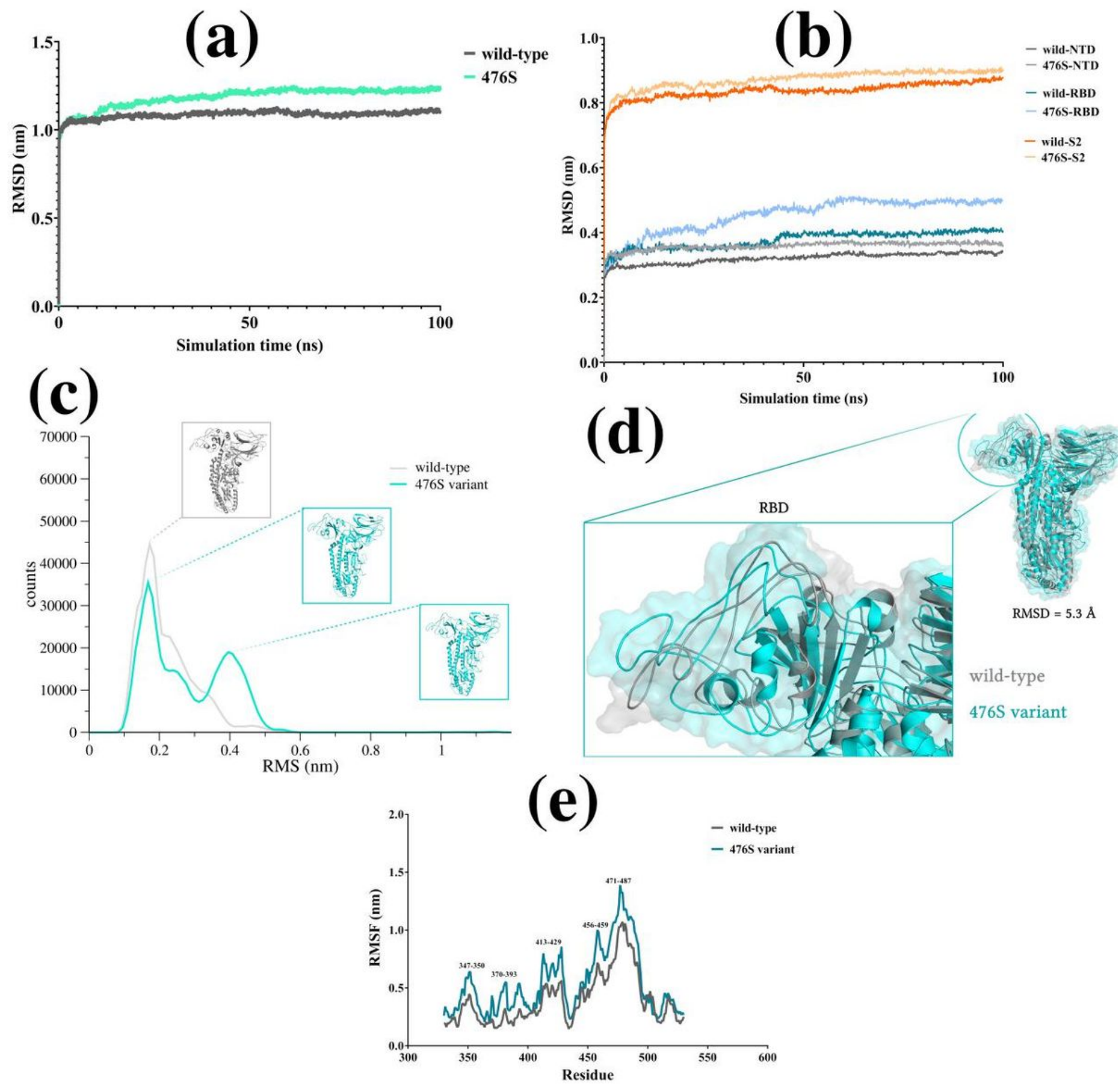

\section{Figure 3}

Impact of the G476S mutation on the structural dynamics of the S-protein. 

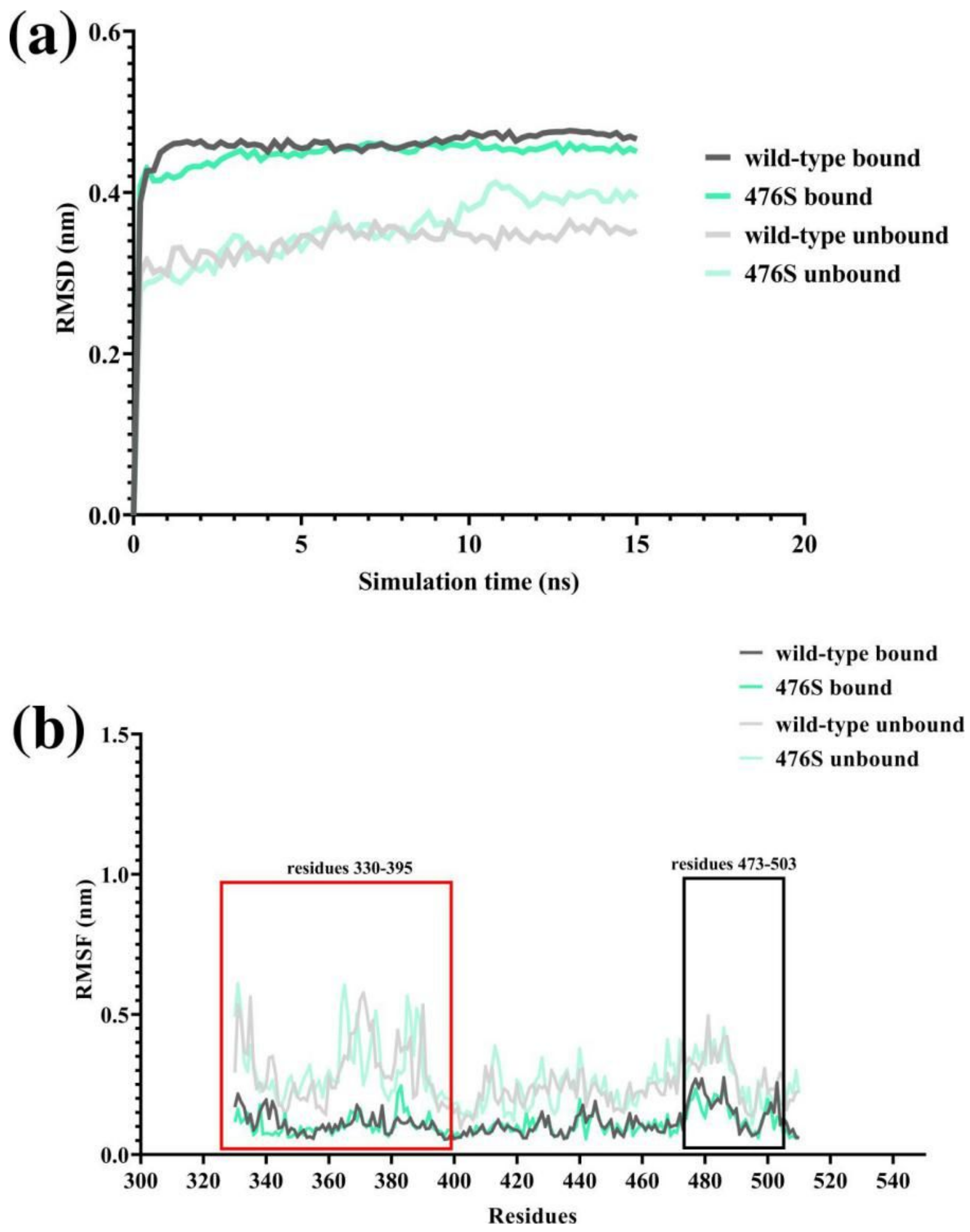

Figure 4

The molecular dynamics of RBD upon binding to the human ACE-2 receptor. (a) The evolution of RBD RMSD for both hACE-2 bound and unbound states (b) Residue-specific flexibility of the RBD for both hACE- 2 bound and unbound states. The most affected and least affected regions are placed in red and black rectangles, respectively. 

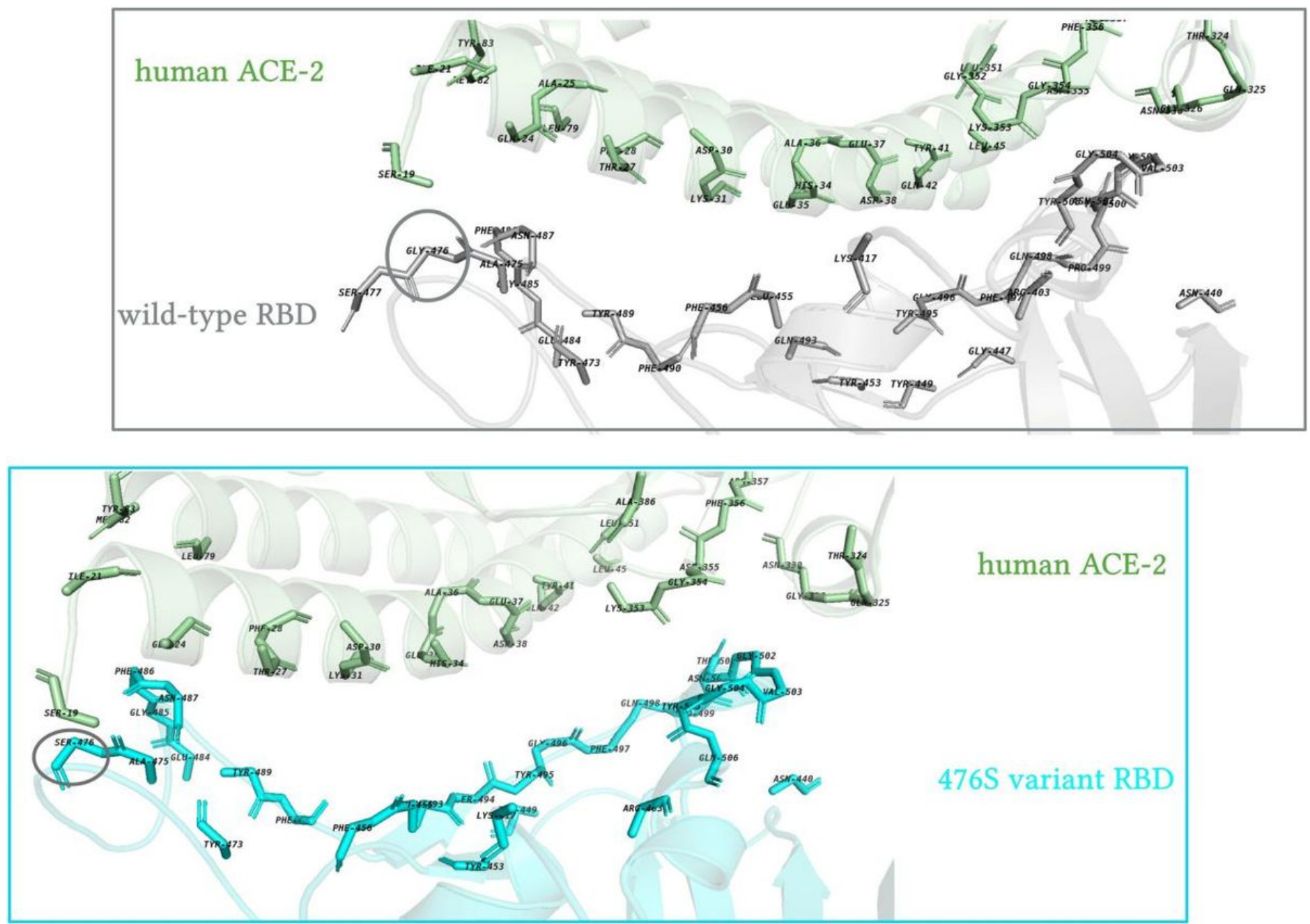

\section{Figure 5}

The interacting interface of the complex showing the amino acids within $5 \AA$ of each protomer. Gly-476 and Ser-476 have been circled. The interface was produced from the middle-structure of the most populated cluster group. 


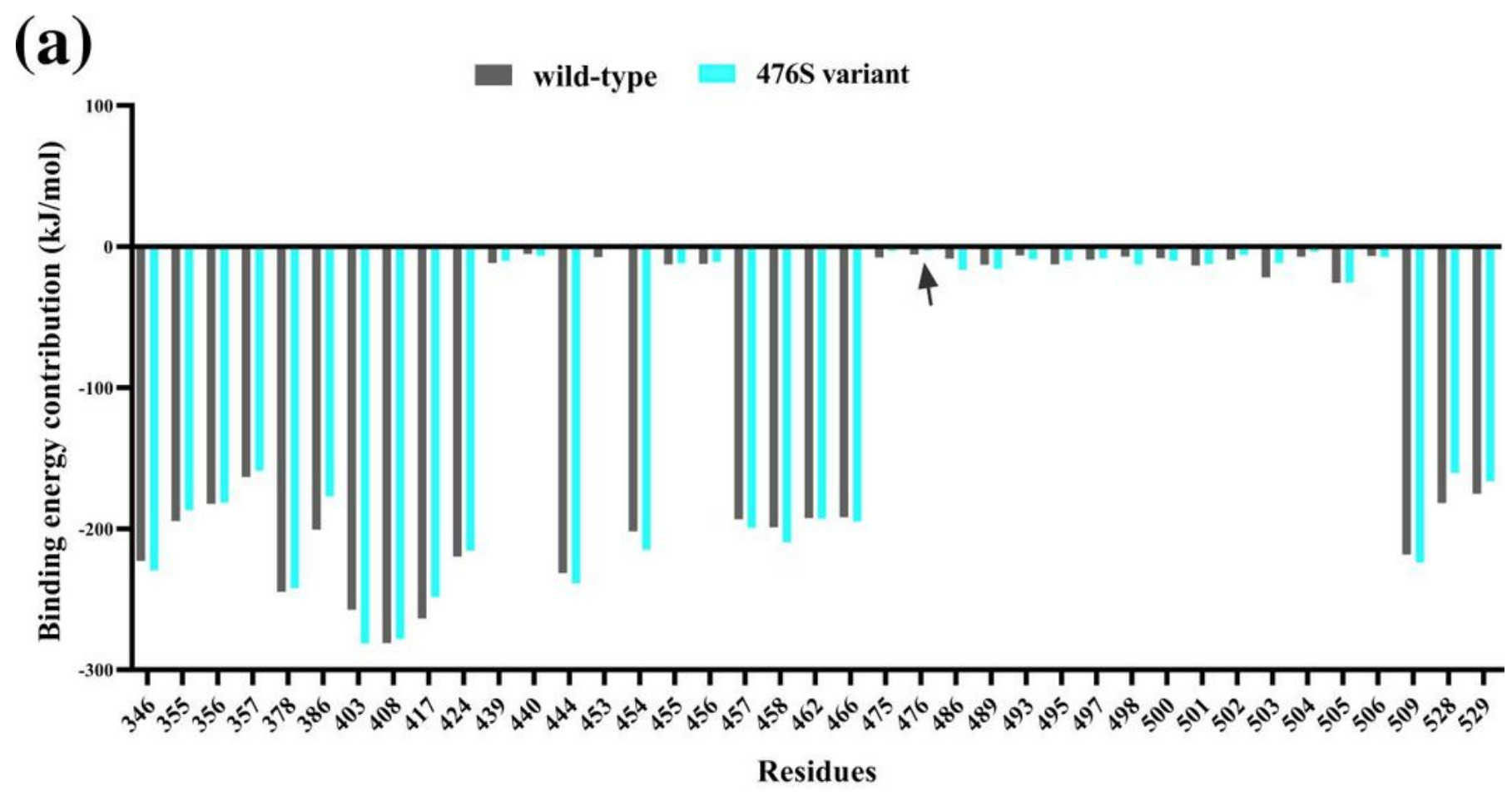

(b)

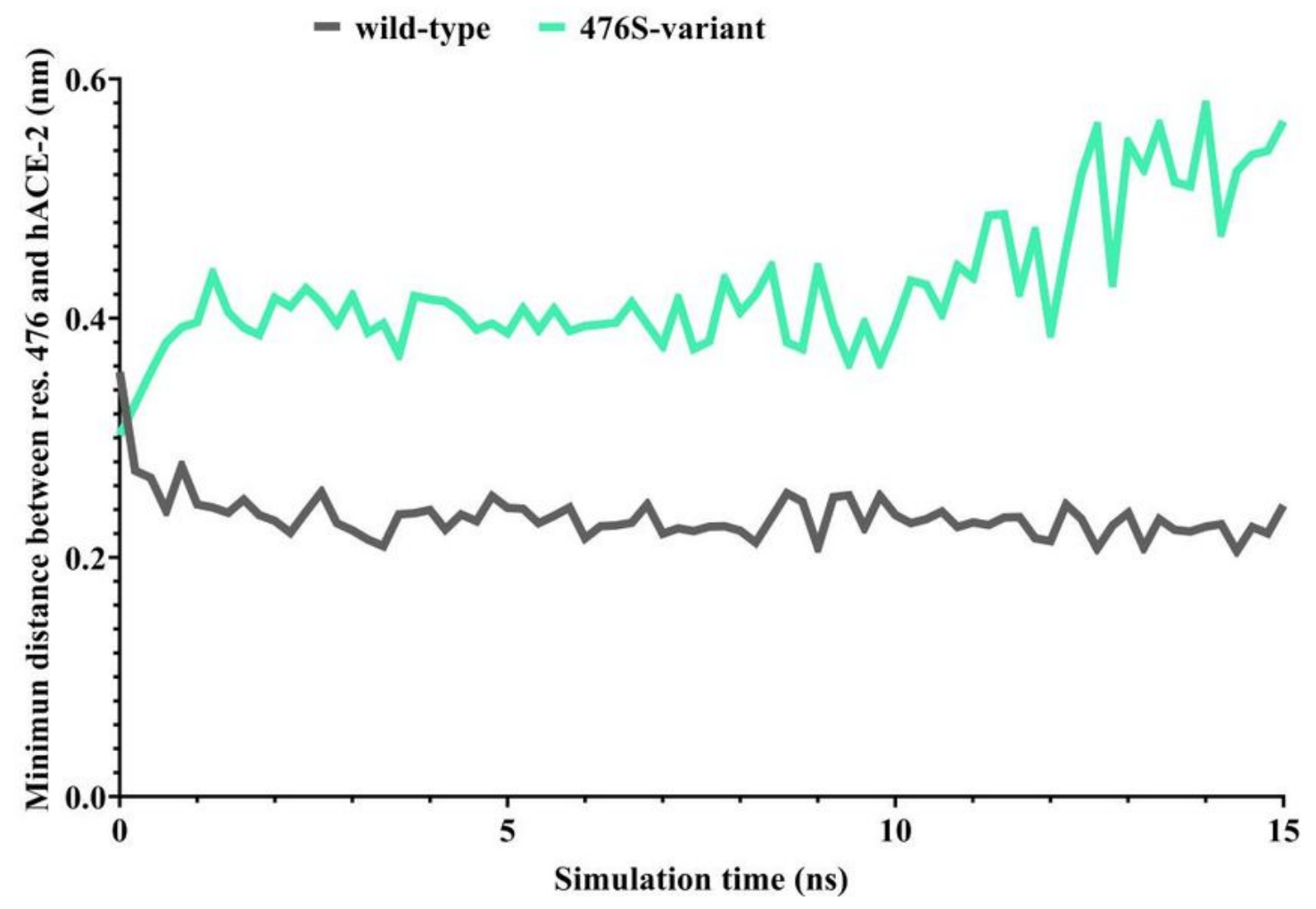

Figure 6

The interactions between RBD and hACE-2. (a) Residue contribution of the RBD residues to the stabilization of the RBD-hACE-2 complex. The contributions from Gly-476 and Ser-476 have been marked on the plot. (b) The minimum distance between Gly476S/Ser-476S and hACE-2. 

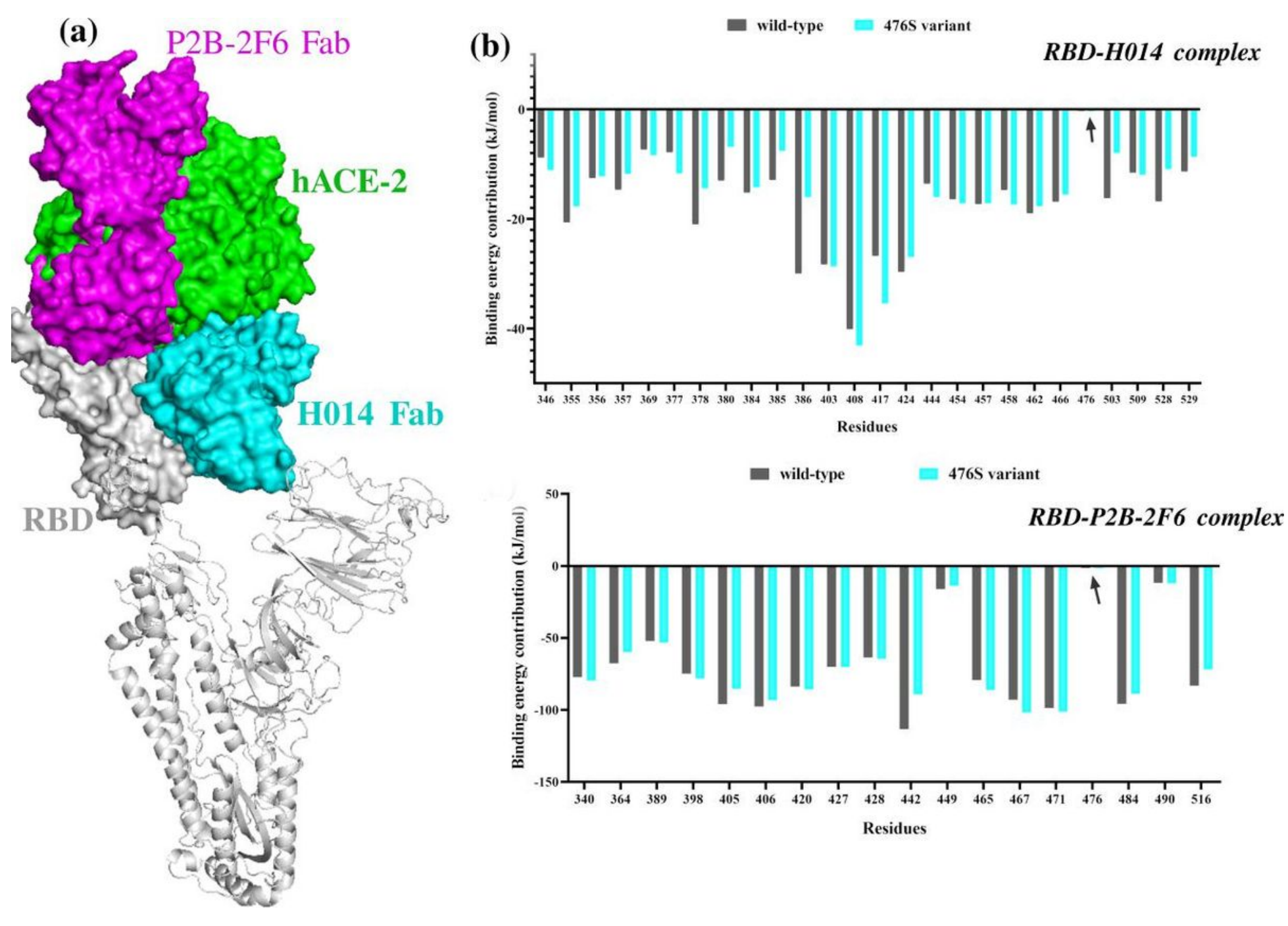

Figure 7

The interaction between SARS-CoV-2 RBD and neutralizing antibodies. (a) Molecular view of the antibody neutralization via sterical clashing or direct competition with RBD for hACE-2 binding (b) The residue contribution of the RBD residues to the stabilization of the RBD-H014 and RBD-P2B-2F6 complexes. The contributions from Gly-476 and Ser-476 have been marked on the plots. 